\title{
Segmentation and texture analysis with multimodel inference for the automatic detection of exudates in early diabetic retinopathy
}

\author{
Jack Lee, Benny Zee*, Qing Li
}

Division of Biostatistics, JC School of Public Health and Primary Care, The Chinese University of Hong Kong, Hong Kong, China Email: ${ }^{\text {bzee@cuhk.edu.hk }}$

Received 10 January 2013; revised 15 February 2013; accepted 2 March 2013

\begin{abstract}
Diabetic retinopathy (DR) is an eye disease caused by the increase of insulin in blood and may cause blindness if not treated at an early stage. Exudates are the primary sign of DR. Currently there is no fully automated method to detect exudates in the literature and it would be useful in large scale screening if fully automatic method is available. In this paper we developed a novel method to detect exudates that based on interactions between texture analysis and segmentation with mathematical morphological technique by using multimodel inference. The texture analysis involves three components: they are statistical texture analysis, high order spectra analysis, and fractal analysis. The performance of the proposed method is assessed by the sensitivity, specificity and accuracy using the public data DIARETDB1. Our results show that the sensitivity, specificity and accuracy are $95.7 \%, 97.6 \%$ and $98.7 \%(\mathrm{SE}=0.01)$, respectively. It is shown that the proposed method can be run automatically and also improve the accuracy of exudates detection significantly over most of the previous methods.
\end{abstract}

Keywords: Texture Analysis; Multimodel Inference; Morphological Technique; Exudates; Diabetic Retinopathy

\section{INTRODUCTION}

Diabetic retinopathy is a severe and common eye disease which can be regarded as a manifestation of diabetes on the retina. It is a major public health problem and diabetic-related eye diseases are the commonest cause of vision defects and blindness in the world. The appearance of microaneurysms, hemorrhages and exudates represent to certain extent the degree of severity of the disease. Exudates are lipid leaks from blood vessels of

"Corresponding author. abnormal retinas and are one of the most prevalent lesions at the early stages of DR [1]. From visual inspection, exudates appear to be yellowish or white color with varying sizes, shape and locations. Automatic exudates detection can assist ophthalmologists in the prevention and allowing the disease to be treated more efficiently.

Many techniques have been employed to perform exudates detection. A complete comparative analysis of the most recent techniques is made on [2]. For these techniques, contextual information was used with Computer-aided detection systems. The context is based on the spatial relation with surrounding anatomical landmarks and similar lesions [3]. Fuzzy C-means clustering was incorporated with spatial neighbourhood information [4]. Mathematical morphologic methods have been used $[5,6]$. Neural Networks and Support-vector machines were used in [7]. Split-and-merge techniques, where region candidates are detected using a combination of coarse and fine segmentation were used in [8]. An approach based on Fisher's linear discriminant analysis making use of color information was introduced in [9]. Naïve-Bayes and Support Vector Machines classifiers were employed in [10]. M. U. Akram et al. [11] proposed a technique that uses filter banks to extract the candidate regions for possible exudates. It also applied a Bayesian classifier as a combination of Gaussian functions to detect exudate and non-exudates regions. Among these techniques, as mentioned in [7], some of methods may incorrectly detect artifacts as exudates especially those appear to be like exudates, or only limit to work well in LUV color space with even illumination image, i.e., the accuracy is low for the case of uneven illumination image. Others may produce either high misclassified portion for images that do not contain exudates (low specificity), or fails to detect faint exudates (low sensitivity).

In order to improve the performance of exudates detection, we developed a method that based on the interactions between texture analysis and segmentation using morphological reconstruction. The texture analysis 
mainly applies on the extracted vessels and enhanced gray level images. It is used for complementing the limitation of exudates segmentation with mathematical morphology approach.

\section{MATERIALS AND METHODS}

The proposed method is designed to detect exudates from retinal images under an automatic platform. It mainly includes four main stages: First, a color retinal image is given as the input and is preprocessed by local contrast enhancement and non-uniform background correction. i.e., the color image is converted to the green component and is preprocessed to normalize and smooth the image and then locate the optic disc (OD) (Section 2.1.2). In the second stage, we eliminate the OD and carry out the exudates detection using mathematical morphology approach (Section 2.2.1). The segmentation of exudates is obtained in pixels and their features such as status of exudates(s), minimal and maximum values corresponding to the aspect ratios, eccentricity, area in pixels representing the size of exudates and the ratio of circularity are also obtained. These features are used to filter out some spurious exudates candidates. We denoted this set of features by $I_{e}$. The third stage is to generate another set of features that come from texture analysis and denoted it by $I_{t}$ (Section 2.2.2). In the final stage, we combined all of the above features to search for significant factors that highly associated with exudates (Section 2.2.3). At this stage, dimension reduction and model selection with multi-inference statistical techniques are applied. Besides, a validation of classifying Exudates/ Non-exudates is given by using both logistic regression and Multi-layer neural network. The proposed block diagram for exudates detection is given in Appendix (Figure A1).

\subsection{Preprocessing and Optic Disc Localization}

The purpose of preprocessing and contrast enhancement is to remove any false artifacts that occur during retinal image acquisition process.

\subsubsection{Preprocessing and Contrast Enhancement}

Since differences in luminosity, contrast, and brightness among different retinal images contribute to the difficulty to extract useful retinal features to distinguish exudates from other bright features in the images, shade correction and noise removal are crucial tasks to prepare the images before processing. To correct uneven illumination of images, a morphological top-hat operator with disk-shaped structuring element and fixed radius of 25 pixels was applied to the green component of the color image. In general, morphological top-hat operator can remove background noise efficiently, which is defined as following.

$$
T H_{o}(G)=G-(G \circ B)
$$

where,

$G=$ original image,

$B=$ structuring element

$(G \circ B)=$ opening operation to $G$ by $B$.

To reduce noise, a $3 \times 3$ (estimated by experimenting) adaptive median filter is applied to the shade corrected image. Its purpose is mainly for the removing impulse noise, smoothing of other noise and reducing distortion, like excessive thinning or thickening of object boundary. The detailed algorithm is given in $[12,13]$.

For the further contrast enhancement, we applied a related operation called "edge enhancement" or "unsharp masking" (UM) to make edges in an image slightly sharper and crisper. The idea of unsharp masking is to subtract a scaled "unsharp" version of the image from the original. In practice, we can achieve this effect by subtracting a scaled blurred image from the original [14]. Figure 1 illustrates the preprocessing results.

\subsubsection{Optic Disc Localization}

The optic disc is a component on the fundus photo from where optic nerves and blood vessels originated. Localization of an optic disc is a vital step in the automated retinal image screening system. The optic disc is exemplified by the largest high contrast among circular shape areas. Referring to the work of U. S. Akram et al. [15], to locate and segment the OD, we can use image averaging and Hough transformation, respectively. The detailed work for OD segmentation is given in [15]. After the OD is localized, we will remove it after we have segmented all exudates candidates involving the OD.

\subsection{Candidate Region Detection and Features Extraction}

After the preprocessing for contrast enhancement, we would remove potential artifacts and enhance the contrast of bright lesions by suppressing and smoothing out all dark and red components present in the retinal image. We then find candidate regions of exudates and gene-

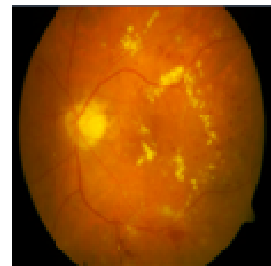

(a)

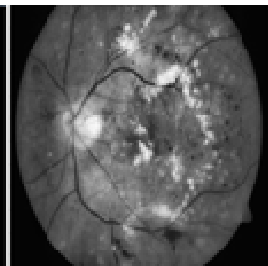

(b)

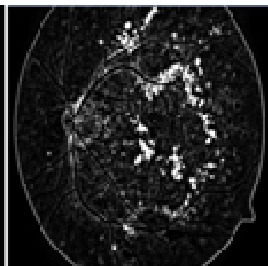

(c)
Figure 1. Result of preprocessing methods. (a) Original image; (b) Filtered image; (c) Enhanced image. 
rate all possible features related to exudates.

\subsubsection{Segmentation and Feature Set 1 Formulation}

Exudates detection using mathematical morphology, FCM, a combination of FCM and mathematical morphology, naïve Bayesian classifier, SVMs and some other techniques have been used. In this paper we apply the mathematical morphology with three different levels of adapted threshold values (applying 90\% confidence interval for Otsu-approach threshold [16]).

We apply the morphological method to eliminate high contrast vessels by using a closing operator before a local variation operator. To ensure that all the neighboring pixels are included in the candidate region, a dilation operator is also applied. Finally the exudates detection areas are obtained by applying a threshold operator to the difference between the original image and the reconstructed image. Finally, the OD is eliminated from the above candidate region(s) image. An example can be seen in Figure 2 (An illustration of the OD localization and removal of OD region is also given in this figure).

The candidate exudates region detection phase extracts as many possible regions as it possibly can for potential exudates. The threshold value is deliberately kept low so that not a single pixel containing exudates will be missed at this stage. We removed the spurious pixels or nonexudates regions in classification stage.

Exudates appear as bright yellow spots with variable size and shape but they have strong and sharp edges. The candidate exudates region extraction stage gives all possible regions that can be considered as potential exudates. If a retinal image I contains $\mathrm{k}$ potential candidate regions, then the set representation for an image $\mathrm{I}$ is $I_{e}$, = $\left\{v_{1}, v_{2}, \ldots, v_{k}\right\}$. For an automated system to distinguish between exudates and non-exudates regions, a feature set is formed for each candidate region. Each object or candidate exudates region is considered as a sample for classification and represented by a feature vector containing $\mathrm{m}$ features; i.e., for a sample candidate region $v$ from an image $\mathrm{I}$, the feature vector is $v=\left\{x_{1}, x_{2}, x_{3}, \ldots\right.$, $\left.x_{m}\right\}$. The description of features we used for classification of exudates and non-exudates regions are as follows:

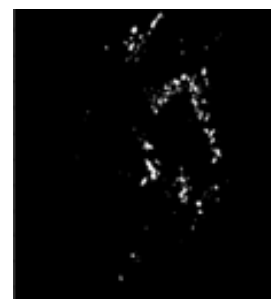

(a)

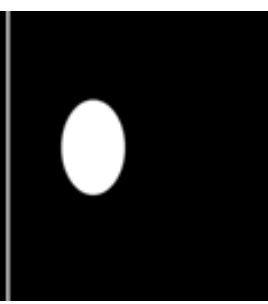

(b)

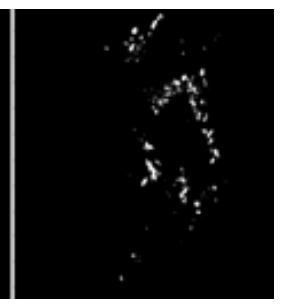

(c)
Figure 2. Result of exudates detected methods. (a) Candidates; (b) OD localized; (c) Exudates.
1) Area $\left(x_{1}\right)$ is the number of pixels in candidate exudates region and is defined as $A=\sum_{v_{i}} 1$ sum of all pixels
in candidate region $v_{i}$.

2) Compactness $\left(x_{2}\right)$ is the measure of shape defined as $C=p^{2} /(4 \pi \mathrm{A})$, where $\mathrm{p}$ and $\mathrm{A}$ are the perimeter and area of candidate region, respectively.

3) "Eccentricity" $\left(x_{3}\right)$ - Scalar that specifies the eccentricity of the ellipse that has the same second-moments as the region. The eccentricity is the ratio of the distance between the foci of the ellipse and its major axis length. The value is between 0 and 1 . ( 0 and 1 are degenerate cases; an ellipse whose eccentricity is 0 is actually a circle, while an ellipse whose eccentricity is 1 is a line segment.)

4) Aspect ratio $\left(x_{4}\right)$ of an image describes the proportional relationship between its width and its height (in rectangle shape).

For our study, we formulated two subgroup features sets: features set 1 is generated from exudates segmentation and features set 2 is generated from texture analysis (see next section). Here in features set 1 we have four features from the segmented candidate exudates region and defined as:

$$
\begin{aligned}
& v=\left\{x_{i j}\right\} ; i=1, \cdots, K ; j=1, \cdots, m \\
& v_{\text {minj }}=\min \left\{x_{i j} ; i=1, \cdots, K\right\} ; j=1,2, \cdots, 4 \\
& v_{\text {maxj }}=\max \left\{x_{i j} ; i=1, \cdots, K ;\right\} ; j=1,2, \cdots, 4
\end{aligned}
$$

$K$ is varied but $m(=4)$ is fixed. Therefore, there are 9 variables from the segmented exudates candidates for each threshold of every image ( 8 from above features and one from the status of exudates). Overall, we generated a total of 27 features from three thresholds of each image, i.e., $I_{e}=\left\{e_{1}, e_{2}, \ldots, e_{27}\right\}$.

Other information of features will be obtained from the approach of texture analysis. Such approach will cover information of features from exudates candidate region such as Mean hue, Mean saturation, Mean value; Mean gradient magnitude for edge pixels and related entropy values [11]. Moreover, some complement information will also be generated from whole image instead of extracted exudates candidate regions since we are not guarantee (surely) to extract all exudates candidates without missing.

Other features (features set 2) from the texture analysis for each image are given from both segmented blood vessels and enhanced gray level image.

\subsubsection{Texture Analysis and Feature Set 2 Formulation}

There are three approaches from texture analysis: 1) Statistical texture analysis involving gray level co-occurrence matrix (GLCM) and run-length matrix; 2) HOS analysis; and 3) fractal analysis to generate some useful 
features from the enhanced image. There are a total of 75 features generated initially. It includes 50 features coming from HOS analysis and 17 features generated from statistical texture analysis, and 8 features (three from mono-spectrum and five from multi-spectrum) from fractal analysis. The following are discussion of methods from the approach of statistical texture analysis, HOS and fractal analysis.

\section{1) Statistical based texture analysis for image pro- cessing}

All related texture features from the statistical-based texture analysis were generated from each retinal image that derived from gray level co-occurrence matrix (GLCM) and run-length matrix [17-25].

For an image of size $M \times N$, the gray level co-occurrence matrix (GLCM) is defined as in [19]

$$
\begin{aligned}
& C_{d}(i, j)=\mid\{(p, q),(p+\Delta x, q+\Delta y): \\
& I(p, q)=i, I(p+\Delta x, q+\Delta y)=j\} \mid
\end{aligned}
$$

where $(p, q),(p+\Delta x, q+\Delta y) \in M \times N, d=(\Delta x, \Delta y)$ and |. denotes the cardinality of a set. Given a gray level $i$ in an image, the probability that the gray level of a pixel at a $(\Delta x, \Delta y)$ distance away is $j$ is

$$
P_{d}(i, j)=C_{d}(i, j) / \sum C_{d}(i, j)
$$

From each co-occurrence matrix we computed the following features:

$$
\begin{aligned}
& \text { Energy: } \sqrt{\text { Angular Second Moment }}=\sqrt{\sum_{i} \sum_{j} P_{d}^{2}(i, j)} \\
& \text { Entropy: } E n=\sum_{i} \sum_{j} P(i, j) \cdot \ln P_{d}(i, j) \\
& \text { Contrast: } \sum_{i} \sum_{j}(i, j)^{2} P_{d}(i, j) \\
& \text { Homogeneity: } \sum_{i} \sum_{j} \frac{1}{1+(i, j)^{2}} P_{d}(i, j)
\end{aligned}
$$

Energy measures the textural uniformity of the image, i.e., the repetition of pixel pairs. Basically it is just the measurement of the denseness or order in the image. Entropy measures disorder or randomness of the image and it is an indication of the complexity within an image, thus, more complex images have higher entropy values. Contrast is a measure of the local variations present (or differences in the GLCM) in the image, so higher contrast values indicate large local variations. Homogeneity (also called an inverse difference moment) is inversely proportional to contrast at constant energy. Similarly at constant contrast, homogeneity is inversely proportional to energy (Park, Lawrence, Windham, Chen, \& Chao, 2002). It measures how close the distribution of elements in the GLCM is to the diagonal of GLCM.

Other measurements such as Moments $1-4$ are defined as:

$$
m_{g}=\sum_{i} \sum_{j}(i, j)^{g} P_{d}(i, j)
$$

where $g$ is the integer power exponent that defines the moment order. Moments are the statistical expectation of certain power functions of a random variable and are characterized as follows [19]: moment 1 is the mean which is the average of pixel values in an image [20]; moment 2 is the standard deviation; moment 3 measures the degree of asymmetry in the distribution; and moment 4 measures the relative peakedness or flatness of a distribution and is also known as kurtosis [21].

Other similar approach can also be used: having the GLCM normalized, we can then derive eight second order statistic features which are also known as haralick features [22] for each image, which are: contrast, correlation, energy, entropy, homogeneity, dissimilarity, inverse difference momentum, maximum probability. In addition to these features, we also applied correlation, dissimilarity, inverse difference momentum and maximum probability, which is different from above mentioned features.

The grey level run-length matrix (RLM) is defined as the numbers of runs with pixels of gray level $i$ and run length $j$ for a given direction [23]. RLMs was generated for each sample image segment having directions $\left(0^{\circ}, 45^{\circ}\right.$, $\left.90^{\circ} \& 135^{\circ}\right)$, then the following five statistical features were derived: short run emphasis, long run emphasis, gray level non-uniformity, run length non-uniformity and run percentage. Basically it allows extraction of higher order statistical texture features. These five measures related method from run-length matrixes of $\theta=0^{\circ}, 45^{\circ}$, $90^{\circ}$, and $135^{\circ}$ were also provided in [25].

\section{2) High order spectra analysis (HOS) for image processing}

Higher order spectra (HOS) are known to have the ability to detect non-linearity and deviations from Gaussianity. Motivated by these, a set of HOS based parameters were proposed as features to differentiate the exudates, and non-exudates. HOS are spectral representations of higher moments and they are derived from the averaged Fourier spectrum signal. The bispectrum $B\left(f_{1}, f_{2}\right)$, of a signal is the Fourier transform (FT) of the third order correlation of the signal. It is given by

$$
B\left(f_{1}, f_{2}\right)=E\left[X\left(f_{1}\right) X\left(f_{2}\right) X *\left(f_{1}+f_{2}\right)\right]
$$

where $X(f)$ is the FT of the signal $x(n T), *$ represents complex conjugation and $E[$.$] stands for the expectation$ operation. It retains Fourier phase information. The frequency f may be normalized by the Nyquist frequency to be between 0 and 1. The bispectrum, given by Equation (6), is a complex-valued function of two frequencies. The bispectrum which is the product of three Fourier coefficients exhibits symmetry and need only be computed in a non-redundant region. Assuming that there is no bispec- 
tral aliasing, the bispectrum of a real-valued signal is uniquely defined with the triangle $0 \leq f 2 \leq f 1 \leq f 1+f 2 \leq$ 1 . This is termed as $\Omega$, the principal domain or the nonredundant region (See the triangle region in Figure A2 in Appendix).

Briefly, HOS based and spectral based features are:

Mean of spectral magnitude for HOS [26]:

$$
M_{\text {ave }}=\frac{1}{L} \sum_{\Omega}\left|B\left(f_{1}, f_{2}\right)\right|
$$

where $B\left(f_{1}, f_{2}\right)$ is the bispectrum of the signal.

Other features such as Entropy 1, 2 and 3 are defined as:

Entropy 1: $P_{1}=-\sum_{k} p_{k} \log p_{k}$

where $p_{k}=\frac{\left|B\left(f_{1}, f_{2}\right)\right|}{\sum_{\Omega}\left|B\left(f_{1}, f_{2}\right)\right|}$, and

Entropy 2: $\quad P_{2}=-\sum_{i} q_{i} \log q_{i}$

where $q_{i}=\frac{\left|B\left(f_{1}, f_{2}\right)\right|^{2}}{\sum_{\Omega}\left|B\left(f_{1}, f_{2}\right)\right|^{2}}$ for HOS.

Entropy 3: $P_{2}=-\sum_{n} r_{n} \log r_{n}$

where $r_{n}=\frac{\left|B\left(f_{1}, f_{2}\right)\right|^{3}}{\sum_{\Omega}\left|B\left(f_{1}, f_{2}\right)\right|^{3}}$ for HOS.

And the feature of bispectrum phase entropy (EntPh):

$$
E n t P h=\sum_{n} p\left(\psi_{n}\right) \log \left(\psi_{n}\right)
$$

where

$$
=\left\{\phi \mid-\pi+\frac{2 \pi n}{N} \leq \phi<-\pi+\frac{2 \pi(n+1)}{N}, n=0,1, \cdots, N-1\right\}
$$

and

$$
\begin{aligned}
& p\left(\psi_{n}\right)=\frac{1}{L} \sum_{\Omega} I\left(\phi\left(b\left(f_{1}, f_{2}\right)\right)\right) \in \psi_{n}, \\
& I(\cdot) \text { is indicator function }
\end{aligned}
$$

$\phi:$ Bispectrum phase angle

$L$ : Number of points within the samples

We used each of these bispectral invariant features for every $18^{\circ}$ from $0^{\circ}$ to $180^{\circ}$. Therefore we obtained total 50 features of HOS. In the next section we will discuss the model-based methods such as fractal model approach. The fractal model is useful for modeling certain natural textures that have a statistical quality of roughness at different scales, and also for texture analysis and discrimination.

\section{3) Fractal analysis}

Fractal model (analysis) may be considered as the model-based texture analysis. We can classify it into mono-fractal and multi-fractal. Since the surface complexity is fundamental to several properties and physical phenomena of a pattern. The diversified complexity of fractal may be described with the concept of fractal dimension [27], which may easily describe the incompleteness or fragmentation of an entirety. Moreover, recent studies show that such surface complexity of image may be described not only by its fractal dimension but also its multifractal spectra, which its mathematical description of a surface can accurately reflect its features, and can be compatible with the various theoretical models which are related to surface structures. Thus we can apply fractal analysis to determine the surface complexity which is measured on the gray scale and by using multifractal spectra one can obtain more detailed information than is possible with the fractal dimension alone. Recently this kind of technique is widely used in retinal vessels analysis.

Measuring fractal dimension has previously attempted to quantify small changes to the human retinal vasculature, not immediately apparent by human observation, and act as an early marker of disease [28-30]. Monofractal analysis is an indicator of vascular change or disease has achieved limited success as retinal vessels may have different properties in different regions and different characteristics can be found depending on the location or scale of the measurement considered. Greater success has been reported by considering the retinal vascular pattern to be geometrical multi-fractal characterized by a hierarchy of exponents rather than a single fractal dimension [31].

We first applied box-counting algorithm (with Hausdorff dimension) approach to calculate fractal dimension in binary type of image (segmentation of vessels) and then the Fourier fractal dimension (FFD) approach, which have been proposed by [28-30]. FFD has been used to quantify the grayscale images projected on to 3-D fractal surface [31]. The advantage of FFD is that it computes the fractal dimension of gray scale images, and eliminates the need for image segmentation [31]. It has also been found to be relatively insensitive to noise and it is believed to work effectively with data having low signal-to-noise ratio $[31,32]$. We adapted the similar FFD approach proposed by Azemin M. and et al. [30]. The parameters related such as slope and intercept are generated based on this approach. Finally we applied the multifractal spectra since it can describe the evolution of the probability distribution of fractal structures [28, 31-33]. Instead of using simple fractals (or monofractals), multifractals are characterized by a hierarchy of exponents, rather than a single fractal dimension. In this paper the box-counting method was used to characterize multiple spectra [31]. 
Based on the above three approaches, the feature set 2 formulated from the texture analysis is given as

$$
I_{t}=\left\{\tau_{1}, \tau_{2}, \cdots, \tau_{n}\right\}
$$

where $n$ is the number of features set 2 for each image. Total features generated from each image are defined as

$$
I=\left\{I_{t}, I_{e}\right\}=\left\{f_{1}, f_{2}, \cdots, f_{n}, \cdots, f_{n+8}, \cdots, f_{n+27}\right\} .
$$

Since $n$ is 75 and so we have a total of 102 features used for classifying the exudates and non-exudates.

\subsubsection{Dimension Reduction and Best Model Searching}

Since there are a total of 102 related variables (factors) for the analysis, but the number of samples is limited thus we need to reduce the dimension (filtering out some not-as-useful factors). This will help us find a more suitable diagnostic model in order to detect exudates with more efficiency. The following are the two major steps.

\section{1) Dimension reduction}

We first reduce dimension since most of the features are correlated or redundant in the model. A penalized logistic regression method (Pelora) [35] was used to cluster the datasets from the above procedures combining all 102 parameters (factors). This is a supervised clustering algorithm that has been used with external information about response variables for clustering genes in genetic study. This algorithm is mainly based on penalize logistic regression analysis and it combines feature selection, supervision, feature clustering and sample classification in a single step. We have adapted this method for features selection generated from retinal images and then classified the variables (potential factors) obtained. This approach will preserve the property of the classified (identified) groups with complex interactions.

\section{2) Model selection with multimodel inference}

After the dimensions have been reduced, we applied the method of automated model selection and modelaveraging that provides a wrapper for GLM and similar functions, automatically generating all possible models with the specified response of exudates(s) and explanatory variables, and determine the best models with a defined criterion (e.g. AIC or AICc). The best model is mainly based on the bias-variance trade-off.

Statistical models are probability density functions, where we can maximize the likelihood $\hat{\theta}=\operatorname{argmax}_{\theta}[f(\cdot, \theta)]$ by minimize the Kullback-Leibler distance from the true model to the approximating model, i.e.,

$$
\hat{\theta} \underset{a . s .}{\longrightarrow} \operatorname{argmax}_{\theta}[K L(g, f(\cdot, \theta))]
$$

Here $K L$ distance is one way to measure the distance between two densities:

$$
K L(g, f(\cdot, \theta))=\int g(y) \log \frac{g(y)}{f(y, \theta)} \mathrm{d} y
$$

$g(y)$ for the discrepancy is weighted by the probability observing the data $\mathrm{y}$, and $\frac{g(y)}{f(y, \theta)}$ is the ratio that measures the discrepancy between the two models for data $y$. AIC selects the model closest to the true model on average. Since the true distribution of $g$ is unknown, so we replace it with the empirical (observed) distribution. Thus the estimation of $K L$ distance is introduced and a bias will also be given. To determine the expected value of this bias, the so called Takeuchi Information Criterion (TIC) is used. Finally under the assumption of approximate model correct, we derived AIC and related criteria.

On the other hands, since AIC selects the model closest to the true model on average (Atypical datasets have lower influence than typical ones) to deal with the model selection uncertainty and usual sampling uncertainty. To do this, we bootstrap the data and produce the distribution of best models. Then apply the smoothed model weights and finally determine: 1) the value of the parameter on average (also its variance) for solving the model selection uncertainty problem; and 2) the standard error for a given model that follows a $\hat{\theta}$ distribution (conditional variance of the estimator), this is to deal with usual sampling uncertainty. Thus we can combine the above two variances to form the overall variance for the estimator $\hat{\theta}$. Such approach reduces the spurious estimates (false positive) problem, which standard model selection techniques often encountered (see Freedman's paradox).

Because algorithm convergence for the best model is not guaranteed and the number of models is large when involving interactions in model, we used a genetic algorithm (better when the number of models to explore is prohibitively large). The model AICc values and complexity are obtained for the selection of the best model. We then applied GLMULTI, an R package for automated model selection and multi-model inference with GLM and related functions. The basic idea of this approach is that from a list of explanatory variables, GLMULTI builds all possible models involving these variables and, optionally, included their pairwise interactions (for the computing of simplicity and easy interpretation reasons we will not consider higher order interaction in this paper). Restrictions can be specified for candidate models, by excluding specific terms, enforcing marginality, or controlling model complexity. Detailed information is referred to [36-39].

The model selection (variable selection in multiple regression framework) framework is given in Figure 3. 


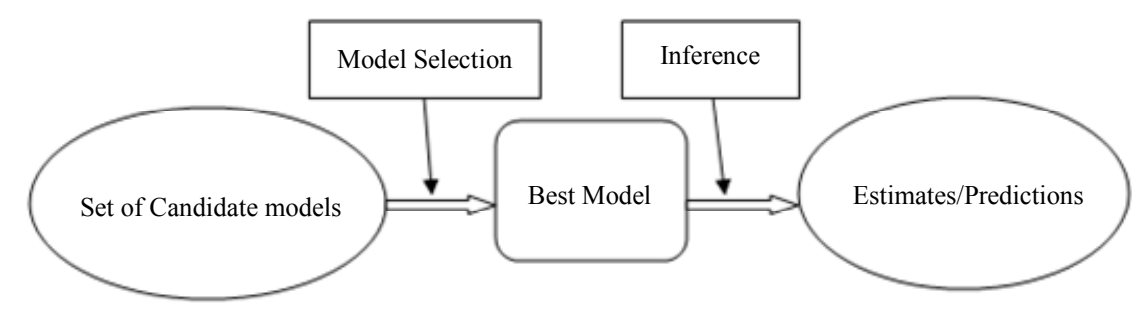

Figure 3. Multi-model inference approach diagram.

\subsubsection{Classification and Validation}

Two different classification methods were investigated to detect exudates. We first applied logistic regression with ROC to classify and validate the abnormal of DR with exudates. Then we applied the approach of Multilayer perception (MLP), a multilayer feed-forward network which is an important class of NNs that can represent non-linear functional mapping between a set of input variables and a set of output variables [40,41]. A MLP with enough units in a single hidden layer can approximate any function, provided the activation function of the neurons satisfies some general constraints $[42,43]$. From these considerations, we decide to use a MLP with one hidden layer, for which the optimum number of hidden neurons was experimentally determined. As neuron activation function in the hidden layer, we chose the hyperbolic tangent sigmoid function (tan-sigmoid) an antisymmetric function in the interval $(-1,1)$. Tan-sigmoid satisfies the constraints in $[42,43]$. Moreover, it improves the learning speed of MLP [40]. In the output layer, we used the logistic sigmoid activation function, that also satisfies the aforementioned constraints and whose outputs lie in the range $(0,1)$. This choice was motivated by the fact of interpreting the outputs of the network as posterior probabilities [41].

The problem of training a NN can be formulated in terms of the minimization of an error function. The choice of a suitable error function and minimization algorithm can improve the performance of MLP. It has been demonstrated [41] that a cross-entropy error function simplifies the optimization process when the logistic sigmoid activation function is used in the output layer. Therefore, we considered this function as an appropriate choice in our study.

Regarding the minimization algorithm, several choices are available for MLP. We selected the scaled conjugate gradients algorithm, for which the error function is guaranteed not to increase during training [41]. Moreover, it generally shows faster convergence when compared to gradient descent-based techniques or even conventional conjugate gradient algorithms [41].

To avoid overfitting and improve generalization, we can also employ a weight decay regularizer [41]. The regularization parameter, $v$, was experimentally settled. If the output is above 0.5 for some input vector, the pro- bability of the image region represented by that input vector of being an EX is greater than that of being a non-EX. Therefore, the output threshold was set to 0.5 .

\section{RESULTS AND DISCUSSION}

Many experiments have been performed on normal and abnormal retinal images based on presenting of exudates. To test and validate our proposed method, the following 89 images from the DIARETDB1 database of resolution $1500 \times 1152$ with their clinician marked images [44] were used to validate our method at the pixel level. 47 of these images contain exudates while the remaining 42 either contain other type of lesions or are normal. We used this database to measure the accuracy of the proposed method based on its ability to distinguish between normal and abnormal images. An example of the classification with MLP is shown in Table 1 (with AUC = 0.98).

The performance of the proposed approach for exudates detection was assessed quantitatively by comparing the results of extractions with the ophthalmologist's ground-truth images. The comparison for the results of the proposed approach (classified using logistic regression and MLP in SPSS) and other previous methods are shown in Table 2. In classification stage, MLP method is used and a training set was obtained from approximately $70 \%$ of whole dataset randomly. The remaining about $30 \%$ will be used for testing. The similar procedure was repeat used in 50 times. The average of measured correctness obtained as the final results.

\section{CONCLUSION}

We propose a novel method to automatically detect exudates from retinal images. The method combined both exudates segmentation and the analysis using statistical texture features, higher order spectra related features and fractal analysis related features. For the segmentation process, we also generate few (four) segmented region properties including maximum of Eccentricity, minimum and maximum of aspect ratio, and also the area of exudates in pixels. For the texture analysis, we generated some multifractal spectrum related features and also some related interaction. We may consider fitting generalized linear models with L1(lasso) and/or L2(ridge) pe- 
Table 1. Example of classification.

\begin{tabular}{lcccc}
\hline \multirow{5}{*}{ Sample } & Classification \\
\cline { 3 - 5 } & Observed & \multicolumn{3}{c}{ Predicted } \\
\cline { 2 - 5 } Training & 0 & 28 & 1 & $96.6 \%$ \\
& 1 & 1 & 34 & $97.1 \%$ \\
& Overall Percent & $45.3 \%$ & $54.7 \%$ & $96.9 \%$ \\
\multirow{2}{*}{ Testing } & 0 & 13 & 0 & $100.0 \%$ \\
& 1 & 0 & 12 & $100.0 \%$ \\
& Overall Percent & $52.0 \%$ & $48.0 \%$ & $100.0 \%$ \\
\hline
\end{tabular}

Dependent variable: Exudates.

Table 2. Comparison of exudates detection methods.

\begin{tabular}{cccc}
\hline \multirow{2}{*}{$\begin{array}{c}\text { Method/Reference } \\
\text { (using DIARETDB1) }\end{array}$} & AUC (\%) & Sensitivity & Specificity \\
\cline { 2 - 4 } & ----- & 86 & 98 \\
Kande et al. [4] & ----- & 70.48 & 98.84 \\
Welfer et al. [5] & 99.4 & 89.3 & 99.3 \\
Jaafar et al. [8] & \multicolumn{3}{c}{ Validation } \\
Our proposed method & 100 & 100 & 100 \\
$\quad$ LR classifier & $(97.8,94.2)$ & $(95.8,91)$ & 98.45 \\
MPLNN classifier & (Using other database) & \\
Sopharak et al. [10] & 98.41 & 92.28 & 98.52 \\
Garcia et al. [7] & 97 & 88.14 & 92.6 \\
Ravishankar et al. [6] & ----- & 94.16 & 91.1 \\
\hline
\end{tabular}

${ }^{a}$ Sensitivity and specificity are given in the form of (training, testing).

nalizes combined with bootstrap technique for feature selection involving Higher order interaction $(>2)$ in future. Overall, the application of multi-inference approach statistical model increased both sensitivity and specificity. For instance, our proposed model without pair-wise interaction, the results of sensitivity, specificity and AUC are $0.915,0.857$ and 0.94 , respectively (used logistic regression classifier). The final result with interaction terms is dramatically improved for the detection of exudates from retinal images. However, despite its superior performance and ability to deal with variant image quality, the proposed method still need to be examined in a large amount of retinal images and also expanded to include all signs of DR. Thus, our future work will undertake detecting the other type of lesions associated with DR.

\section{REFERENCES}

[1] Fong, D.S. et al., (2003) Diabetic retinopathy. Diabetes Care, 26, 226-229. doi:10.2337/diacare.26.1.226

[2] Sopharak, A., Uyyanonvara, B., Barman, S. and Willia- son, T. (2010) Comparative analysis of automatic exudate detection algorithms. WCE, London.

[3] Sánchez, C.I., et al. (2010) Improving hard exudates detection in retinal images through a combination of local and contextual information. 2010 IEEE International Symposium on Biomedical Imaging: From Nano to Macro, Rotterdam, 14-17 April 2010, 5-8.

[4] Kande, G., Subbaiah, P. and Savithri, T. (2008) Segmentation of exdates and optic disk in retinal images. IEEE Processing of 6th Indian Conference on Computer Vision, Graphic \& Image, Bhubaneshwar, 16-19 December 2008.

[5] Welfer, D., et al. (2009) A coarse-to-fine strategy for automatically detecting exudates in color eye fundus images. Computerized Medical Imaging and Graphics, 34, 228-235.

[6] Ravishankar, S., Jain, A. and Mittal, A. (2009) Automated feature extraction for early detection of diabetic retinopathy in fundus images. IEEE Conference on Computer Vision and Pattern Recognition (CVPR), Miami, 20-25 June 2009.

[7] Garcia, M., et al. (2009) Neural network based detection of hard exudates in retinal images. Computer Methods and Programs in Biomedicine, 93, 9-19. doi:10.1016/j.cmpb.2008.07.006

[8] Jaafar, H., Nandi, A. and Al-Nuaimy, W. (2010) Automated detection of exudates in retinal images using a split and merge algorithm. EUSIPCO, Aalborg, 23-27 August 2010.

[9] Sanchez, C.I., et al. (2008) A novel automatic image processing algorithm for detection of hard exudates based on retinal image analysis. Medical Engineering \& Physics, 30, 350-357. doi:10.1016/j.medengphy.2007.04.010

[10] Sopharak, A., et al. (2009) Machine learning approach to automatic exudate detection in retinal images from diabetic retinopathy. Journal of Modern Optics, 57, 1-12.

[11] Akram, M.U., et al. (2012) Automated detection of exudates in colored retinal images for diagnosis of diabetic retinopathy. Applied Optics, 51, 4858-4866.

[12] Gonzalez, R.C. and Woods, R.E. (2008) Digital image processing. 3rd Edition, Prentice Hall, Upper Saddle River.

[13] Hwang, H. and Haddad, R.A. (1995) Adoptive median filters: New algorithm and results. IEEE Transactions on Image Processing, 4, 499, doi:10.1109/83.370679

[14] Tanaka, G., Suetake, N. and Uchino, E. (2010) Image enhancement based on nonlinear smoothing and sharpening for noisy images. Journal of Advanced Computational Intelligence and Intelligent Informatics (JACIII), 14, 200207.

[15] Akram, M.U., Khan, A., Iqbal, K. and Butt, W.H. (2010) Retinal image: Optic disk localization and detection. Image Analysis and Recognition, Lecture Notes in Computer Science, 6112, 40-49. doi:10.1007/978-3-642-13775-4_5

[16] Otsu, N. (1979) A threshold selection method from graylevel histograms. IEEE Transactions on Systems, Man, and Cybernetics, 9, 62-66. doi:10.1109/TSMC.1979.4310076 
[17] Tan, J.H., Ng, E.Y.K. and Acharya, U.R. (2009) Study of normal ocular thermogram using textural parameters. Infrared Physics \& Technology, 53, 120-126.

[18] Bremananth, R., Nithya, B. and Saipriya, R. (2009) Wood species recognition system using GLCM and correlation. International Conference on Advances in Recent Technologies in Communication and Computing (ARTCom' 09), Kottayam, 27-28 October 2009, 615-619.

[19] Bailey, R.R. and Srinath, M.D. (2002) Orthogonal moment features for use with parametric and non-parametric classifiers. IEEE Transactions on Pattern Analysis and Machine Intelligence, 18, 389-399.

[20] Silakari, S., Motwani, M. and Maheshwari, M. (2009) Color image clustering using block truncation algorithm. International Journal of Computer Science Issues, 4, 3135.

[21] Press, W.H., Flannery, B.P., Teukolsky, S.A. and Vetterling, W.T. (1990) Numerical recipes in C: The art of scientific computing. Cambridge University Press, New York, 1990.

[22] Acharaya, U.R., Ng, E.Y.K., Tan, J.H., Sree, S.V. and Ng, K.H. (2012) An integrated index for the identification of diabetic retinopathy stages using texture parameters. Journal of Medical Systems, 36, 2011-2020.

[23] Tuceryan, M. and Jain, A.K. (1993) Texture analysis. In: Chen, C.H., Pau, L.F. and Wang, P.S.P., Eds., Handbook of Pattern Recognition \& Computer Vision, World Scientific Pub Co Inc., Singapore City. doi:10.1142/9789814343138 0010

[24] Weszka, J.S. and Rosenfield, A. (1976) An application of texture analysis to material inspection. Pattern Recognition, 8, 195-200. doi:10.1016/0031-3203(76)90039-X

[25] Galloway, M.M. (1975) Texture classification using gray level run length. Computer Graphics and Image Processing, 4, 172-179. doi:10.1016/S0146-664X(75)80008-6

[26] Acharya, U.R., Chua, K.C., Ng, E.Y.K., Wei, W. and Chee, C. (2008) Application of higher order spectra for the identification of diabetes retinopathy stages. Journal of Medical Systems, 32, 431-488.

[27] Hubbard, L.D., Brothers, R.J., King, W.N., Clegg, L.X., Klein, R., Cooper, L.S., Sharrett, A.R., Davis, M.D. and Cai, J. (1999) Methods for evaluation of retinal microvascular abnormalities associated with hypertension/ sclerosis in the atherosclerosis risk in communities study. Ophthalmology, 106, 2269-2280. doi:10.1016/S0161-6420(99)90525-0

[28] Azemin, M.Z.C., Kumar, D.K., Wong, T.Y., Wang, J.J., Kawasaki, R. and Mitchell, P. (2010) Retinal stroke prediction using logistic-based fusion of multiscale fractal analysis. 2010 IEEE International Conference on Imaging Systems and Techniques (IST), Thessaloniki, 1-2 July 2010, 125-128.

[29] Azemin, M.Z.C., Kumar, D.K., Wong, T.Y., Wang, J.J., Kawasaki, R., Mitchell, P. and Arjunan, S.P. (2010) Fu- sion of multiscale wavelet-based fractal analysis on retina image for stroke prediction. 2010 Annual International Conference of the IEEE Engineering in Medicine and Biology Society (EMBC), Buenos Aires, 31 August 2010-4 September 2010, 4308-4311,

[30] Azemin, M.Z.C., Kumar, D.K., Wong, T.Y., Kawasaki, R., Mitchell, P. and Wang, J.J. (2011) Robust methodology for fractal analysis of the retinal vasculature. IEEE Transactions on Medical Imaging, $\mathbf{3 0}$.

[31] Stosic, T. and Stosic, B.D. (2006) Multifractal analysis of human retinal vessels. IEEE Transactions on Medical Imaging, 25, 1101-1107. doi:10.1109/TMI.2006.879316

[32] MacGillivary, T.J., Patton, N., Doubal, F.N., Graham, C. and Wardlaw, J.M. (2007) Fractal analysis of the retinal vascular network in fundus images. Proceedings of the 29th Annual International Conference of the IEEE EMBS, Lyon, 23-26 August 2007

[33] Andjelkovic, J., Zivic, N., Reljin, B., Celebic, V. and Salom I. (2008) Application of multifractal analysis on medical images. Wseas Transactions on Information Science and Applications, $\mathbf{5}$.

[34] Faust, O., Acharya, R., Ng, E.Y.K., Ng, K.H. and Suri, J.S. (2012) Algorithm for the automated detection of diabetic retinopathy using digital fundus images: A review. Journal of Medical Systems, 36, 145-157.

[35] Marcel, D. and Peter, B. (2003) Supervised gene clustering with penalized logistic regression. Research Report No.115, Switzerland, 2003

[36] Anderson, D.R. (2008) Model based inference in the life sciences. Springer-Verlag, New York. doi:10.1007/978-0-387-74075-1

[37] Barton, K. (2009) MuMIn: Multi-model inference. R Package Version 0.12.2/r18. http://R-Forge.R-project.org/projects/mumin/

[38] Buckland, S.T., Burnham, K.P. and Augustin, N.H. (1997) Model selection: An integral part of inference. Biometrics, 53, 603-618. doi:10.2307/2533961

[39] Burnham, K.P. and Anderson, D.R. (2002) Model selection and multimodel inference. Springer-Verlag, New York.

[40] Haykin, S. (1999) Neural networks: A comprehensive foundation. Prentice-Hall International, New Jersey.

[41] Bishop, C.M. (2004) Neural networks for pattern recognition. Oxford University Press, New York.

[42] Hornik, K. (1991) Approximation capabilities of multilayer feedforward networks. Neural Networks, 4, 251-257. doi:10.1016/0893-6080(91)90009-T

[43] Huang, G.B., Chen, Y.Q. and Babri, H.A.(2000) Classification ability of single hidden layer feedforward neural networks. IEEE Transactions on Neural Networks, 11, 799-801. doi: $10.1109 / 72.846750$

[44] DIARETDB1 database (2007).

http://www2.it.lut.fi/project/imageret/diaretdb1/index.htm 1 


\section{APPENDIX}

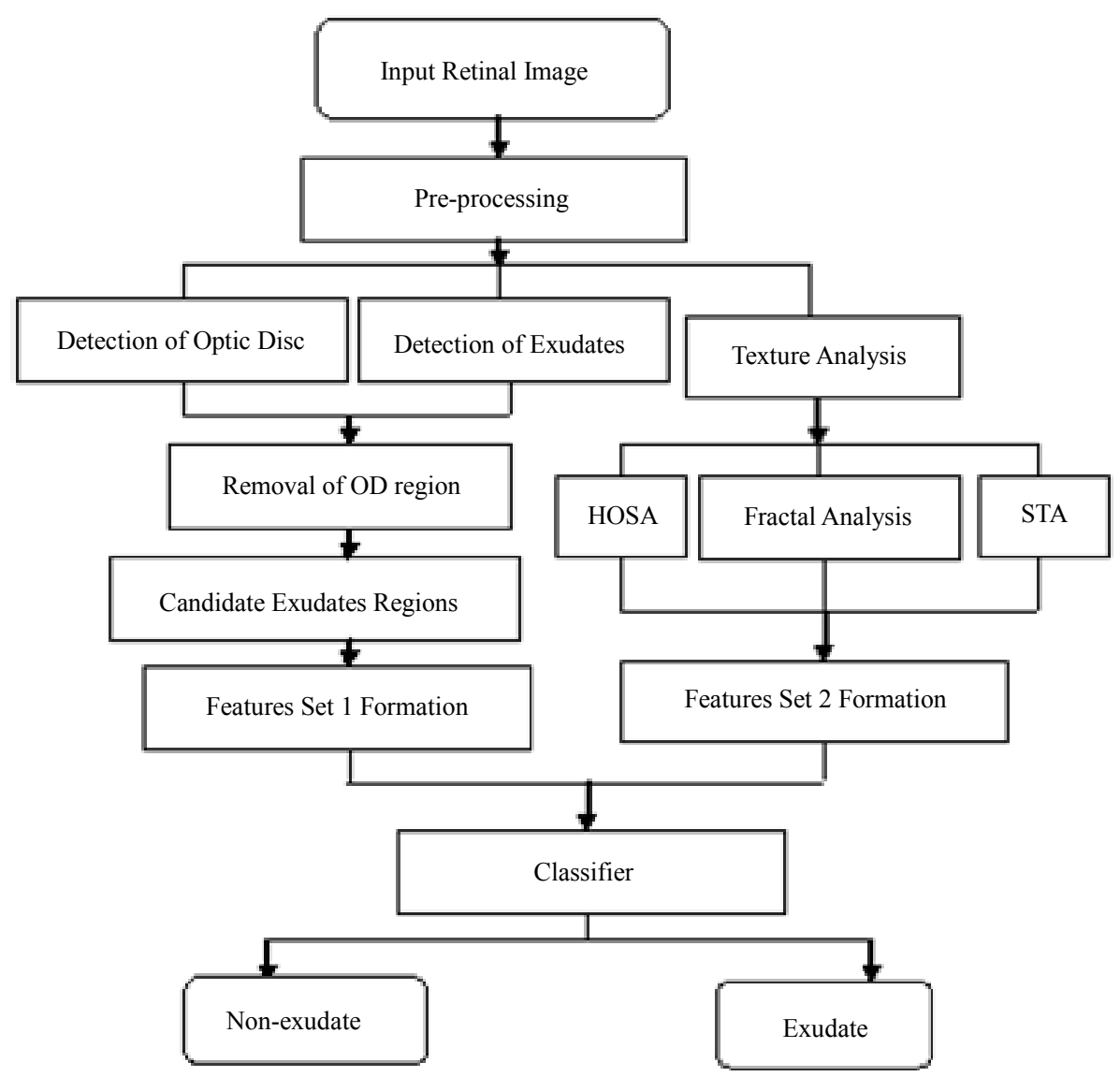

Figure A1. The proposed block diagram for exudates detection.

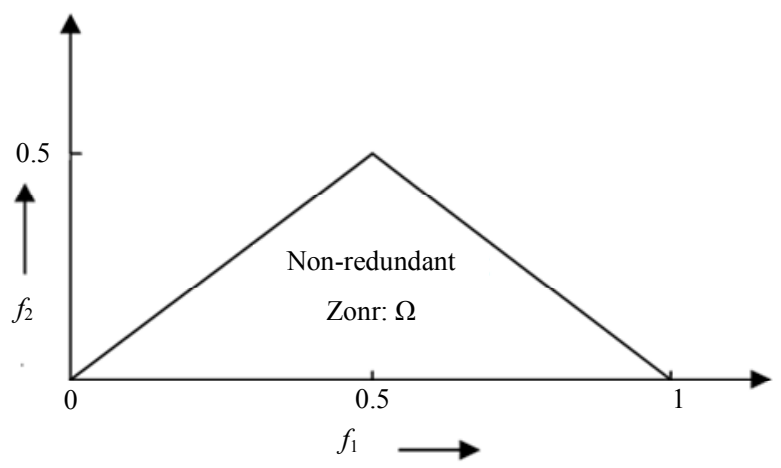

Figure A2. Non-redundant region $(\Omega)$ of computation of the bispectrum for real signals. Parameters are calculated from this region. 